\title{
RADIAL MOTIONS NEAR THE GALACTIC CENTRE AND THE ORIGIN OF GALACTIC SPIRAL STRUCTURE
}

Th. Schmidt-Kaler

Astronomisches Institut der Ruhr-Universität Bochum

The fundamental difficulty of explaining the spiral structure of galaxies by material arms is the winding dilemma which restricts the life-time of a spiral to about one rotation period. This dilemma is resolved by the density wave concept. However, the density waves are damped by various processes. We calculated the life time of the density wave for our Galaxy $\left(\tau=8 \cdot 10^{8}\right.$ yrs) as well as for 24 nearby spiral galaxies with well-known data. It appears that the density wave can explain the existence of spiral arms for only about three rotation periods. After that time the energy of the wave has to be re-supplied. For this, various mechanisms have been proposed, e.g.

1) tidal origin - which requires rather special circumstances

2) resonant interaction - which is energetically insufficient

3) local Jeans-type instability in the outer regions - which also may not be able to provide sufficient energy

4) explosions of the nucleus - which involve high inclinations to the plane.

The fact that no galaxy of low luminosity $M_{v}>-15$ has a nucleus and that there exists no spiral galaxy with $\mathrm{M}_{\mathrm{v}}>-15$ leads us to seek the origin of spiral structure in the nucleus. The expansion motions observed in M31 and in our Galaxy (summarized by Schmidt-Kaler 1973, 1975) are in line with this. New HI-observations confirm the run of the expanding velocities with a maximum near $R=0.8 \mathrm{kpc}$ almost coincident with the inner maximum of the rotation curve (fig. 1). 


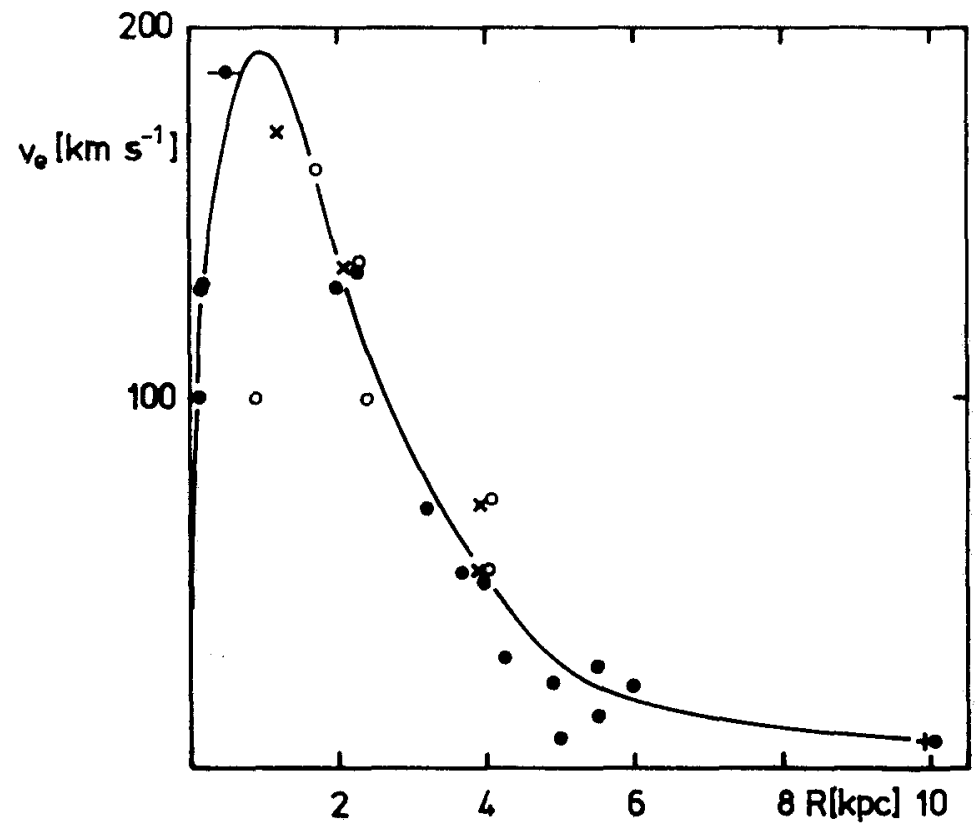

Fig. 1: The expansion curve of our Galaxy. Dots: optical, molecular line and older HI data (Schmidt-Kaler 1973), circles: HI data (Cohen and Davies 1976), crosses: HI features with $|z|<150$ pc (Rohlfs, Schmidt-Kaler 1977), plus-sign: local HI (Yuan 1977).

Transport of energy $\varepsilon$ and momentum $j$ by density waves takes place with the group velocity in the directions

$$
\begin{aligned}
& \left.\mathrm{R}_{i L} \longrightarrow \mathrm{R}_{\mathrm{CO}} \longrightarrow \mathrm{R}_{\mathrm{OL}} \begin{array}{c}
\text { short } \\
\text { (long) }
\end{array}\right\} \quad \text { wave mode } \\
& \varepsilon<0, j<0 \quad \varepsilon>0, j>0
\end{aligned}
$$

where $\mathrm{R}_{i L}, \mathrm{R}_{O L}, \mathrm{R}_{\mathrm{C}}$ resp. are the inner und outer Lindblad and the corotation radius. Since the sign of the wave energy changes at $R$ the short wave mode transports positive energy and momentum outwards throughout the wave region from the inner to the outer Lindblad radius.

Two mechanisms were envisaged:

1) A central mass asymmetry (oval or bar-like distortion of the central spheroid) acting as a motor to drive the density wave (Feldman 
and Lin 1973, Feitzinger and Schmidt-Kaler 1977).

2) Material transport of energy and momentum by mass outflow near $R_{i L}$. Assuming $\dot{M}=0.5 \mathrm{M}_{\odot} / \mathrm{yr}$ we find as supply of kinetic energy

$$
\dot{\mathrm{E}}_{\mathrm{kin}}=\frac{1}{2} \mathrm{v}_{\mathrm{e}}^{2} \dot{\mathrm{M}} \cong 4 \cdot 10^{38} \mathrm{exg} \mathrm{\textrm {s } ^ { - 1 }}
$$

to be compared with the energy loss of the density wave

$$
\frac{E_{d w}}{\tau} \equiv \frac{5 \cdot 10^{54} \mathrm{erg}}{8 \cdot 10^{8} \mathrm{yrs}} \cong 2 \cdot 10^{38} \mathrm{erg} \mathrm{s} \mathrm{s}^{-1},
$$

and as supply of momentum from the central spheroid with radius $R_{s} \cong R_{i L}$

$$
\dot{J}=\frac{1}{2} \dot{M} R_{s}^{2}\left(\omega_{s}-\omega\right) \cong 1 \cdot 10^{54} \mathrm{erg}
$$

to be compared with

$$
\frac{J_{d w}}{\tau}=\frac{E_{d w} /\left(\omega-\omega_{p}\right)}{\tau}=0,5 \cdot 10^{54} \mathrm{erg} .
$$

The oval distortion of the central spheroid can be described by instabilities of classical incompressible fluid models: The mode $\mathrm{m}=1$ is stable (Lyttleton 1953). The first unstable mode $\mathrm{m}=2$ is the Riemann (1860) instability for flattenings $\mathrm{c} / \mathrm{a} \leqq 0.30$ with the critical frequency $w_{c}=1 / 2 w_{s}$ (Bryan 1888). Secular instability of this mode sets in for $\mathrm{c} / \mathrm{a}=0.58$. The higher modes $\mathrm{m}=3,4, \ldots$ become only unstable after $\mathrm{m}=2$ (Chandrasekhar 1969).

The Riemann instability was further studied in three ways:

a) by calculating finite amplitude perturbations after the method of Dirichlet (1860) and Rossner (1967)

b) by a stellar-dynamical description of the hydrodynamic model

c) by calculating the stabilizing influence of a spherical halo or a flat disk (fig. 2). 


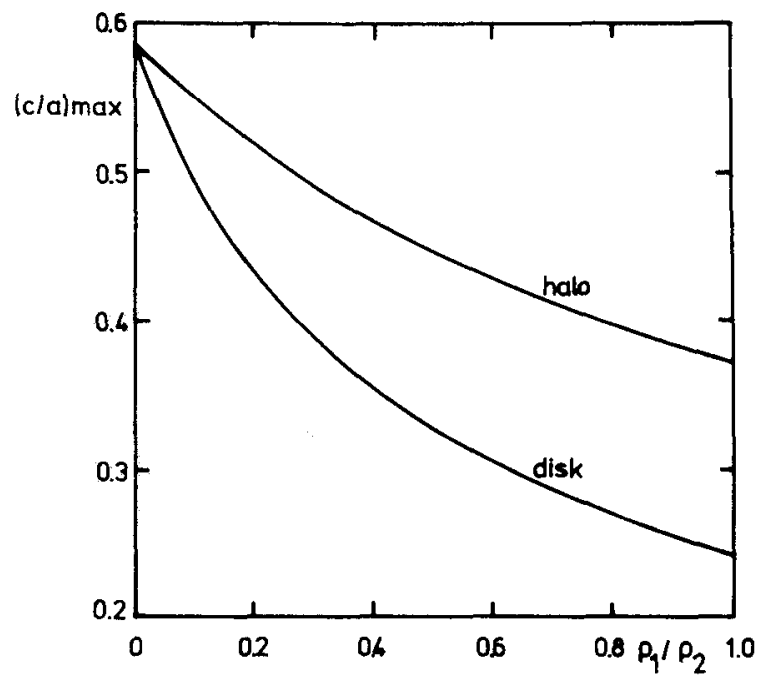

Fig. 2: The stabilizing influence of a spherical halo or a circular flat disk on the Riemann instability of a spheroid as a function of the relative density of the embedding halo.

Calculations of realistic models with various flattenings $c / a=0.13 \ldots 0.75$ (Wiegandt 1977) show that explosions in the nucleus with energies of the order $q=E_{k i n} / U \cong 0,01$ (binding energy $U$ of the central spheroid) lead to $v_{e} \cong 10 \mathrm{~km} \mathrm{~s}$ and $\dot{\mathrm{M}} \cong 0.1 \mathrm{M}_{\circ} / \mathrm{Yr}$. Continuous energy release reduces the required energy by a factor of $10^{1-2}$, restriction to the gas component alone being affected by the explosion to further reduction by a factor $10^{3}$, while viscous forces increase it by a factor $10^{2}$ so that finally an energy release of $10^{54}$ erg on a time scale of $10^{6}$ yrs appears sufficient to explain the basic instability. Outside the central spheroid the gas outflow can be described by a nonspherical galactic wind (focussed by the Riemann wave) with the typical sound velocity $v_{s} \cong 120 \mathrm{~km} \mathrm{~s}^{-1}$ in the spheriodal component (planetary nebulae) $\mathrm{v}_{e^{\prime}} \cdot \max \cong(1-2) \mathrm{v}_{\mathrm{s}} \cdot$

This work is based on the collaboration with Drs. Feitzinger, Quiroga, Rohlfs and Mr. Wiegandt which is gratefully acknowledged. 


\section{REFERENCES}

Bryan, G.H.: 1888, Acta Math. 7. 187.

Chandrasekhar, S.: 1969, Ellipsoidal Figures of Equilibrium, Yale University Press, New Haven.

Cohen, R.J. and Davies, R.D.: 1976, Monthly Notices Roy. Astron. Soc. 175,1 .

Dirichlet, G.L.: 1860, J. reine und angewandte Mathematik 58, 181. Feitzinger, J. and Schmidt-Kaler, Th.: 1977, in preparation.

Feldman, S.I. and Lin, C.C.: 1973, Studies Appl. Math. 52, 1. Lyttleton, R.A.: 1953, The Stability of Rotating Liquid Masses, Cambridge University Press, Cambridge.

Riemann, B.: 1860, Abh. Königl. Ges. Wiss. Göttingen 9, 3. Rohlfs, K., Schmidt-Kaler, Th.: 1977, in preparation.

Rossner, L.F.: 1967, Astrophys. J. 149, 145.

Schmidt-Kaler, Th.: 1973, Vistas in Astronomy, 19, 69.

Schmidt-Kaler, Th.: 1975, Optische Beobachtungsprogramme zur galaktischen struktur und Dynamik, Bochum (Ed.: Schmidt-Kaler, Th.) 75. Wiegandt, R.: 1977, in preparation.

Yuan, Ch.: 1977, private communication. 